\title{
Lesion Detectability in 2D-mammography and Digital Breast Tomosynthesis using Different Targets and Observers
}

\author{
Premkumar Elangovan ${ }^{1,2}$, Alistair Mackenzie ${ }^{2}$, David R. Dance ${ }^{2,3}$, Kenneth C. Young ${ }^{2,3}$ and \\ Kevin Wells ${ }^{1}$ \\ ${ }^{1}$ Medical Imaging Group, Centre for Vision, Speech, and Signal Processing, University of Surrey, \\ Guildford, GU2 7XH, United Kingdom \\ ${ }^{2}$ National Coordination Centre for the Physics of Mammography (NCCPM), Royal Surrey County \\ Hospital, Guildford GU2 7XX, United Kingdom \\ ${ }^{3}$ Department of Physics, University of Surrey, Guildford, GU2 7XH, United Kingdom \\ E-mail: premkumar.elangovan@nhs.net
}

Short title: 2D vs DBT lesion detection 4AFC study

\begin{abstract}
This work investigates the detection performance of specialist and non-specialist observers for different targets in 2D-mammography and digital breast tomosynthesis (DBT) using the OPTIMAM Virtual Clinical Trials (VCT) Toolbox and a 4-alternative forced choice (4AFC) assessment paradigm.

Using 2D-mammography and DBT images of virtual breast phantoms, we compare the detection limits of simple uniform spherical targets and irregular solid masses. Target diameters of $4 \mathrm{~mm}$ and $6 \mathrm{~mm}$ have been chosen to represent target sizes close to the minimum detectable size found in breast screening, across a range of controlled contrast levels. The images were viewed by a set of specialist observers (five medical physicists and six experienced clinical readers) and five non-specialists.

Combined results from both observer groups indicate that DBT has a significantly lower detectable threshold contrast than 2D-mammography for small masses (4mm: $2.1 \%$ [DBT] vs $6.9 \%$ [2D]]; $6 \mathrm{~mm}$ : $0.7 \%$ [DBT] vs $3.9 \%$ [2D]) and spheres (4mm: $2.9 \%$ [DBT] vs $5.3 \%$ [2D]; $6 \mathrm{~mm}: 0.3 \%$ [DBT] vs $2.2 \%[2 \mathrm{D}])(p<0.0001)$. Both observer groups found spheres significantly easier to detect than irregular solid masses for both sizes and modalities $(p<0.0001)$ (except $4 \mathrm{~mm}$ DBT). The detection performances of specialist and non-specialist observers were generally found to be comparable, where each group marginally outperformed the other in particular detection tasks. Within the specialist group, the clinical readers performed better than the medical physicists with irregular masses $(p<0.0001)$.
\end{abstract}

The results indicate that using spherical targets in such studies may produce over-optimistic detection thresholds compared to more complex masses, and that the superiority of DBT for detecting masses over 2D-mammography has been quantified. The results also suggest specialist observers may be supplemented by non-specialist observers (with training) in some types of 4AFC studies. 


\section{Keywords}

Virtual clinical trials, image simulation, 2D-mammography, digital breast tomosynthesis, 4AFC study, non-specialist observers, physicists, radiologists, general population.

\section{Introduction}

Two dimensional X-ray mammography has been adopted as the standard imaging method for use in national breast screening programmes for early breast cancer detection (Karim-Kos et al 2008, Hevie et al 2014). However, a major shortcoming is the limited ability of observers to detect lesions due to superposition of tissue. To address this, digital breast tomosynthesis (DBT) has been introduced and deployed alongside 2D-mammography in some early adopter breast assessment centres (Gilbert et al 2016, Ciatto et al 2013). Studies have indicated that DBT substantially improves the visibility of lesions by suppressing much of the overlying anatomy (Skaane et al 2013, Wallis et al 2012, Niklason et al 1997, Diekmann and Bick 2007). However, DBT requires thorough evaluation in clinically relevant conditions before widespread adoption in routine breast screening.

Clinical trials are the conventional approach for such evaluations, but this approach is time-consuming and expensive. As a result, many such evaluations are undertaken on small cohorts, or selected groups of screen-detected cases for side-by-side evaluation (Ciatto et al 2013). In the latter case, this necessarily biases the sample selection of detailed cases. Taken together, these factors act as a brake on the introduction and adoption of new effective screening technologies and techniques. Alternatively, Virtual Clinical Trials (VCTs) can be used to supplement clinical trials using simulation methods (Maidment 2014).

VCTs are conducted using computerized modelling tools which are validated to simulate radiological images comparable to their real clinical counterparts. The images can be synthesised by either inserting simulated cancer pathology (Shaheen et al 2010, Rashidnasab et al 2013a, 2013b) into clinical images (Elangovan et al 2014) or by inserting into a complete simulated breast (Li et al 2009, Bliznakova et al 2003, Graff 2016, Bakic et al 2002a, 2002b, Elangovan et al 2017). The image acquisition process and associated image formation and degradation processes are modelled using specialized tools to mimic the system or technology under consideration (Elangovan et al 2016, Mackenzie et al 2012,2014). These tools are then validated for clinical realism by means of observer studies or quantitative metrics. This is to ensure that the results of VCTs are comparable to those of clinical trials with human subjects. Thus VCTs allow for a rapid evaluation and comparison of various breast imaging modalities (Gong et al 2006).

One type of virtual clinical trial that is widely used to compare the detection performance of breast imaging modalities is the m-alternative forced choice ( $m$-AFC) paradigm (Burgess 1995, Burgess 1999). In $m$-AFC studies, a series of $m$ target or signal detection experiments are presented to the observer. The resulting data from a group of observers are used to quantify the observer detection performance expressed for example as minimum detectable target contrast or size. A number of previous studies have used this approach to compare and evaluate breast imaging modalities. For example, prior work investigated the effect of lesion location, lesion size and beam quality on lesion detection performance (Huda et al 2004, 2005, 2006); the effect of quantum and anatomical noise on microcalcification detection (Lai et al 2010); comparison of various reconstruction techniques (Mie'ville et al 2012) and different medical displays (Rashidnasab et al 2016); and quantified lesion 
and micro-calcification detectability in 2D and DBT simulated (Hadjipanteli et al 2016) and hybrid images (Timberg et al 2012), the latter using simulated lesions inserted into clinical images. In this work, we use the $4 \mathrm{AFC}$ paradigm to compare the lesion detectability in 2D-mammography and DBT systems using 4AFC tasks that are representative of real-world clinical situations.

Previous studies have used spheres as idealised targets to represent mammographic lesions e.g. (Mie'ville et al 2013, Gong et al 2006, Young et al 2013). Psychophysical data on object recognition suggests the human visual system can detect such regular target shapes easily and efficiently, largely regardless of context (Biederman 1987). However, detection and identification of irregularly shaped objects are thought to use a more sophisticated set of detection and recognition processes (Biederman 1987, Shams et al 2002) (and references therein). This may affect the interpreted equivalency of mAFC detection thresholds for spheres and their application to real mass detection in mammography screening.

Prior work has also used medical physicists as observers in isolation (Timberg et al 2012, Gong et al 2006), or mixed with radiologists for alternative forced choice (AFC) studies (Timberg et al 1987, Chakraborty et al 1986). As AFC only involves Signal Known Exactly target recognition, without the specialist task of image search, equivalency between such observers has been assumed. Given a paucity of baseline studies, it is of interest therefore to investigate this assumption, and further, whether non-specialists might also compare favourably to these two observer types in such studies.

Thus, the objective of this work was to use the 4-alternative forced choice (4AFC) paradigm to examine target threshold detectability in 2D-mammography and DBT systems using different targets and different observers. Target sizes were deliberately chosen that straddle the current known limits of detectability (Timberg et al 2012) in 2D-mammography and DBT.

This was accomplished using the OPTIMAM VCT Toolbox (Elangovan et al 2014, 2016) whereby breast models that contain realistic anatomical breast structures (Elangovan et al 2017) and a set of validated synthetic mass lesions (Rashidnasab et al 2013) were used to create detection tasks across a range of target sizes and contrast levels. Validated VCT modelling tools were used to model various image formation and degradation processes for the Hologic Selenia Dimensions 3D system (Hologic Inc., Bedford, Massachusetts, USA) including system geometry, noise, blur and scatter (Elangovan et al 2014). Images were presented to panels of specialist and non-specialist observers in a 4AFC study.

\section{Materials and methods}

\subsection{Breast models}

Four virtual breast models with different tissue distributions and a voxel resolution of $100 \mu \mathrm{m}$ were created for this study. The models were $6 \mathrm{~cm}$ thick with an average glandularity of $20 \%$ by volume. The glandularity was chosen in line with the density measurements performed on a set of real images of $6 \mathrm{~cm}$ thick compressed breasts using the Volpara $^{\mathrm{TM}}$ breast density measurement tool (Volpara Health Technologies Limited, Wellington, New Zealand) (Highnam et al 2010).

The virtual breast models (Elangovan et al 2017) used a biologically inspired approach whereby, features and patterns extracted from DBT images were used to simulate various tissue components. The breast models contain adipose tissue, fibro-glandular tissue, Cooper's ligaments, blood vessels and skin layers. The features were extracted from selected real DBT volumes using a combination of 
methods which included region growing, morphological operations, supervised classification/annotation and 3D spline interpolation. Simulated radiological images of these breast models have been previously validated for use in 4AFC studies (image segments of size $3 \times 3 \mathrm{~cm}^{2}$ ), statistically, by power spectrum analysis and for realism via ROC-based analysis. Figure 1 shows $2 \mathrm{D}-$ mammogram image segments constructed from the breast models chosen for the study.

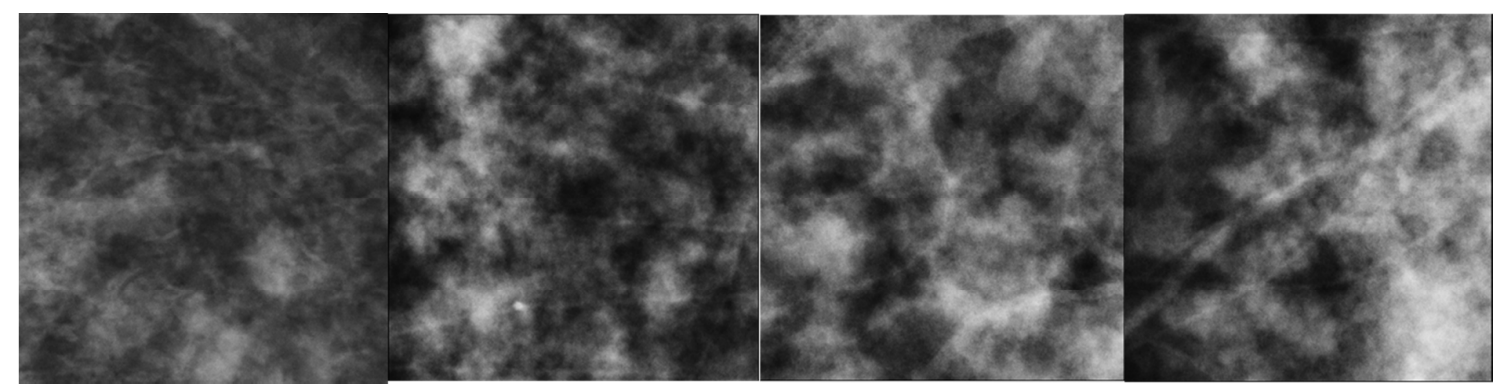

Figure 1

2D-mammogram image segments of four breast models. The images illustrate different texture patterns simulated in each breast model.

\subsection{Irregular solid lesions and spherical targets}

Six simulated mass lesions with similar physiological characteristics (irregularly shaped margins and homogenous texture) but each unique in visual appearance, together with uniform spheres were used as targets.

Irregular solid masses were generated using Diffusion Limited Aggregation (DLA) - a 3D fractal growth method that produces irregular structures. The appearance of the DLA lesions has been previously validated for realism by means of observer studies in 2D (Rashidnasab et al 2013a) and DBT (Rashidnasab et al 2013b). When embedded in a simulated breast background, each mass used had received experienced ( $>20$ years) radiologist observer feedback ratings of 'definitely realistic' in both 2D and DBT.

The mass and sphere target volumes were initially generated at a voxel resolution of $35 \mu \mathrm{m}$ and corresponded to $9 \mathrm{~mm}$ diameter. These volumes were then downscaled to the desired volumes, with nominal diameters of $4 \mathrm{~mm}$ and $6 \mathrm{~mm}$ for the study. Targets were inserted into the breast models by voxel replacement after supersampling the simulated breast tissue volume to $35 \mu \mathrm{m}$ resolution in the vicinity of the insertion.

\subsection{Radiological image simulation framework}

A collection of modelling tools (Elangovan et al 2014) designed to simulate various system acquisition and image degradation processes was used to produce 2D and DBT images of the ROIs containing targets. In this study, we used the system geometry and image acquisition settings for the Hologic Selenia Dimensions 3D system. The attenuation values for different tissue components of the breast model were derived from the elemental compositions of adipose and glandular tissue (Hammerstein et al 1979), whereas, the composition of Cooper's ligaments and blood vessels were assumed to be that of adult skeletal muscle (ICRU 1992, Sandborg et al 2003). Exposure factors (2D: $\mathrm{W} / \mathrm{Rh}, 31 \mathrm{kVp}$; DBT: W/Al, $33 \mathrm{kVp}$ ) for image simulation were set according to those used under automatic exposure control for real breasts of equivalent size and glandularity. An MGD of $2.5 \mathrm{mGy}$ was simulated for 2D and DBT in line with measurements made on a Hologic Selenia Dimensions 3D system for a $6 \mathrm{~cm}$ thick compressed breast (Dance et al 2011). 
A conventional 2D-mammography projection image and 15 DBT projection images were produced using ray tracing (Siddon 1985) for the above X-ray spectrum (Boone et al 1997). The effect of the anti-scatter grid was simulated in the 2D images by applying a grid factor which was determined by the empirical measurements performed on the Hologic system (Elangovan et al 2014). No grid factor was applied to the DBT images. The amount of scatter to be added was derived from a scatter-toprimary ratio look-up table constructed from Monte Carlo simulation data for standard breast models of different thicknesses (Diaz et al 2014) and added to the projections before additional noise and blur were incorporated in the images (Mackenzie et al 2012, 2014). The blur included intrinsic detector blur, sampling blur due to aperture size of pixels, focal spot blur and motion blur caused due to the Xray tube motion in DBT. The noise model simulates a combination of quantum, electronic and structural noise sources in the images. Both 2D-mammography and DBT projections were acquired with a pixel size of $70 \times 70 \mu \mathrm{m}^{2}$. After acquisition, the DBT projections were re-binned to $140 \times 140$ $\mu m^{2}$ pixels. The $2 \mathrm{D}$ image was processed using the manufacturer's image processing tool (Hologic LORAD FFDM Selenia V5.0). DBT planes were produced using the manufacturer's image reconstruction tool which employs a filtered back-projection technique. The processed 2D image had a pixel size of $70 \times 70 \mu \mathrm{m}^{2}$ and reconstructed DBT planes had a pixel size of $100 \times 100 \mu \mathrm{m}^{2}$. The separation between the DBT planes was $1 \mathrm{~mm}$. Figure 2 illustrates the resulting processed 2D and DBT targets embedded in the breast tissue model.
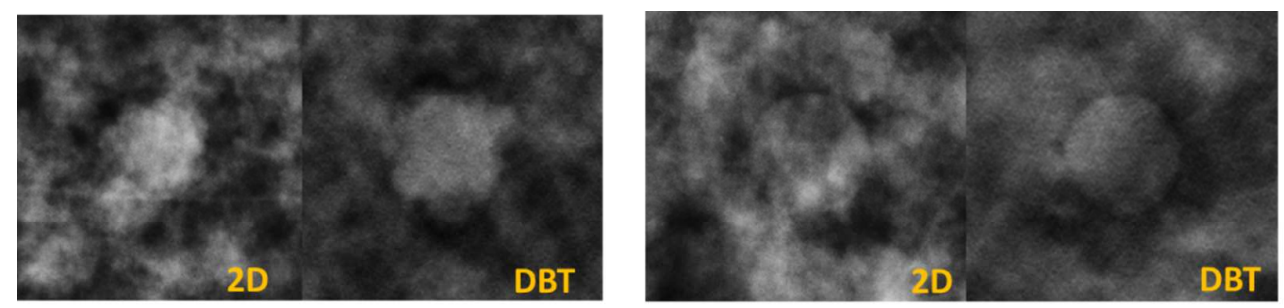

Figure $22 \mathrm{D}$ and DBT image pairs used in the study: lesion target (left) and spherical target (right).

\subsection{Data preparation}

Radiological images containing targets with a range of contrast levels were simulated. The desired contrast was generated purely by changing the location of insertion of the target within a range of breast models. Attenuation properties of the targets were assigned to be same as glandular tissue throughout. For each modality and target type, three contrast levels were chosen to ensure that the $90.7 \%$ detection threshold occurred within the range of results (using prior pilot studies). The contrast is defined as the relative difference between the mean background signal and the mean target signal computed at the insertion site, calculated using raw image data (unprocessed) for 2D images. For DBT data, a 2D raw image with 2D exposure factors was created for the purposes of assessing contrast, so that all contrast measurements (DBT and 2D) are referenced to 2D image contrast measurements. The background pixel intensity was calculated on the pixels occupying a circular region $(8 \mathrm{~mm}$ for $4 \mathrm{~mm}$ lesion, $12 \mathrm{~mm}$ for $6 \mathrm{~mm}$ ) around the target excluding the region occupied by the target.

This metric allows comparison between 2D and DBT with reference to the visibility of lesions in the 2D image. Thus a smaller threshold contrast for DBT than for 2D would mean that lesions at the DBT threshold contrast would be seen with DBT but would not be seen when imaged using the 2D system. 
Therefore our metric allowed a clinically realistic comparison since the primary purpose of the study was to investigate DBT as a potential alternative to $2 \mathrm{D}$ in terms of lesion detection.

For each experimental condition (contrast, modality), 50 image segments containing masses, and 20 image segments containing spheres were simulated resulting in a database of 210 2D and 210 DBT images. It should be noted that some images with $6 \mathrm{~mm}$ spherical targets were simulated at $0 \%$ contrast to present observers with challenging detection tasks. Contrast here refers to average contrast between the object and local ROI background, so that even at very low contrast, pilot studies revealed that observers can detect spheres when only a small section of their sharp regular edge was visible. Table 1 summarizes the experimental conditions simulated.

Table 1 Experimental conditions chosen for the study (SEM on the contrast values is $<2 \mathrm{e}^{-5}$ )

\begin{tabular}{ccccc}
\hline & \multicolumn{2}{c}{ 2D } & DBT \\
\cline { 2 - 5 } & $\begin{array}{c}\text { Average } \\
\text { contrast levels }\end{array}$ & Number of images & $\begin{array}{c}\text { Average } \\
\text { contrast levels }\end{array}$ & Number of images \\
\hline Masses (4mm) & $9 \%, 7 \%, 5 \%$ & 150 & $5 \%, 3.5 \%, 2 \%$ & 150 \\
Spheres (4mm) & $7 \%, 5 \%, 3 \%$ & 60 & $3.5 \%, 2 \%, 1 \%$ & 60 \\
Masses (6mm) & $5 \%, 3 \%, 1 \%$ & 150 & $4 \%, 2.5 \%, 1 \%$ & 150 \\
Spheres (6mm) & $3 \%, 2 \%, 1 \%$ & 60 & $2 \%, 1 \%, 0 \% *$ & 60 \\
No Target & - & 900 & - & 900 \\
& $*$ to achieve an average of 0\% contrast, the object was inserted in a highly glandular region \\
\hline MGD for both 2D and DBT: 2.5mGy
\end{tabular}

\subsection{AFC observers and study conditions}

The 4AFC study was conducted using an in-house Java-based plug-in (ImageJ 1.50i, NIH, USA) as shown in Figure 3. This used the 4AFC convention of presenting a series of signal detection experiments with each trial displaying the target in isolation, located superior to four image quadrants, one of which contained the embedded target (Macmillan and Creelmans 2004). Observers were required to identify the quadrant that most likely contained the target. The breast tissue background for all four quadrants was chosen from the same breast model with equivalent glandularity but at different locations. The quadrant of the image containing the target was randomly shuffled between trials. For 2D images, a 2D projection of the target in each trial was provided as a signal cue. For DBT images, a scrollable slice stack of the target was provided as a signal cue that was synchronised with scrolling of the four DBT quadrants. The central in-focus plane was shown as the initial DBT image out of 12 planes for $4 \mathrm{~mm}$ targets and 18 planes for $6 \mathrm{~mm}$ targets. Multiplanar viewing allowed the observers to better visualise the 3D morphology of the targets mimicking real DBT viewing conditions. The experiments were conducted in low ambient light $(<6 \mathrm{LUX})$ on a high resolution monitor (Barco, B$8500,5 \mathrm{MP}$, Belgium). A concentric toto circle was shown in each quadrant to indicate the potential location of the centralised target.

For the first arm of the study five medical physicists and six clinical readers viewed the images to examine the detection thresholds of masses and spheres. The clinical readers' group included three radiologists, a breast clinician and two radiographers with a clinical screening experience ranging 
between 2 and 18 years with a mean of 7.5 years. The clinical readers' group only participated in the study with $4 \mathrm{~mm}$ targets.
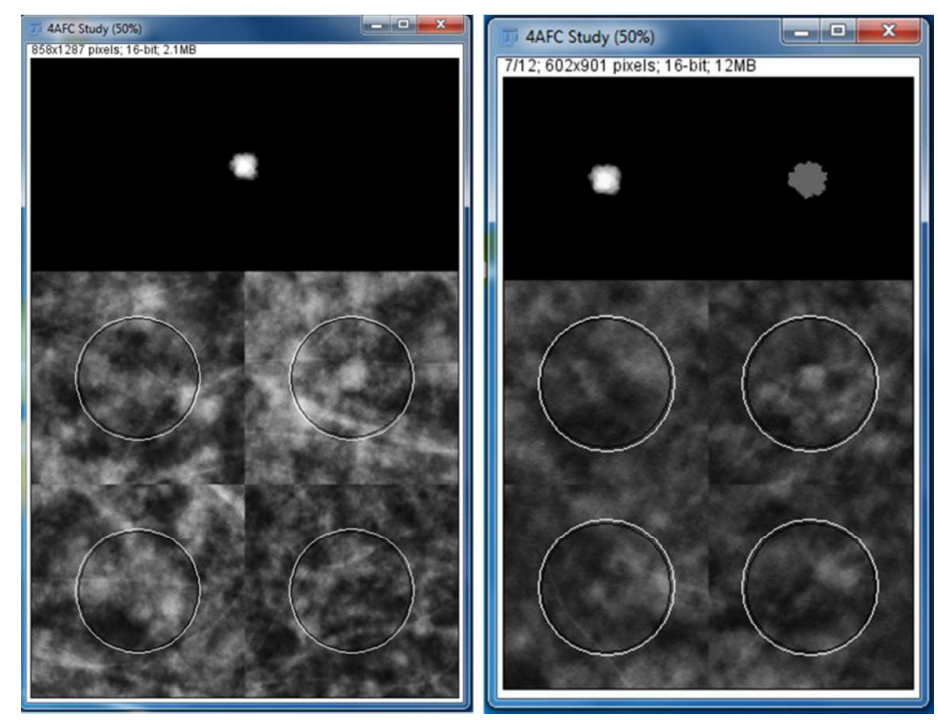

Figure 32 2D and DBT 4AFC study screenshot. 2D projection of the target was provided as a signal cue for the 2D study (left), and in addition, a 3D scrollable slice stack of the target was provided in the DBT study (right).

For the second arm of the study, the above methodology was repeated using a non-specialist observer group. This group included an Undergraduate student, a Masters student, a $\mathrm{PhD}$ research student, a Postdoctoral scientist and a Gynaecologist. The observers' age ranged between 21 and 31 years with a mean of 27 years. None of the non-specialist observers had any prior experience with mammography image data. Prior to the 4AFC study, both the non-specialist observers and clinical readers reviewed a short 4AFC training set comprising 15 2D, and 15 DBT images, to gain feedback and familiarization with the task and the software. The training session lasted approximately 15-20 minutes, and the participants were provided with real-time feedback on their decisions. The specialist group of medical physicists did not undergo any training prior to the study, but had prior experience with mammography image data and had participated in similar studies in the past.

\subsection{Threshold contrast calculation}

Threshold contrast is defined here as the contrast at which the observer makes $90.7 \%$ correct decisions for a particular target (mass or sphere), corresponding to a detectability index d' of 2.5 (Macmillan and Creelmans 2004). This operating point was chosen because of its low coefficient of variation in 4AFC type experiments (Burgess 1995, Burgess 1999). Thus, threshold contrast for 2D-mammography and DBT systems for different targets was determined from the observer responses of both groups.

The results of the study were analysed by pooling the data for each group and converting the percentage correct responses for different target contrasts into equivalent detectability index values. Then, the threshold contrast $(90.7 \%$ correct response or d' of 2.5$)$ was computed from the linear fit between detectability index and target contrasts. The observer responses for each target contrast were bootstrapped 1000 times, and average threshold contrast along with confidence intervals (percentile method) were computed. The threshold contrast values were compared for both 2D and DBT for both target types (spheres and masses). 
A Student t-test was used to calculate the statistical significance of differences in the threshold contrast values between the two observer groups. Finally, the reading times between the two groups were compared for both modalities and types of target.

\section{Results and Discussion}

Figure 4 summarizes the results of the $4 \mathrm{AFC}$ study with error bars showing $95 \%$ confidence intervals. The results include the threshold contrast computed for specialists (clinical readers and medical physicists) and non-specialists separately, and the threshold contrast computed after pooling the data from both observer groups. Note, the plots for the combined observers are not just an arithmetic average of specialist and non-specialist observers; for combined plots, these data were pooled and new fits undertaken and threshold contrasts computed after bootstrapping. Therefore, the combined error bars lie somewhere between specialist and non-specialist observers, but will not necessarily lie exactly at the midpoint. For each modality, target type and size, three data points were available corresponding to the three contrast values used. The target contrast followed a linear relationship with d' as expected (Burgess 1995, Burgess 1999). The reading times for the two groups are also presented in Table 2. The results of the statistical test for both contrast detection thresholds and reading times are shown in Table 3.

The combined threshold contrast for all observers computed for the 4mm lesion in 2D-mammography $(6.9 \%)$ was found to be approximately three times the threshold contrast computed for DBT $(2.1 \%)$. Similarly for the $6 \mathrm{~mm}$ lesion, the combined threshold contrast computed for 2D-mammography (3.9\%) was approximately four times the threshold contrast computed for DBT $(0.7 \%)$. These results show that the threshold contrast for DBT is significantly lower than that of $2 \mathrm{D}$ for both observer groups $(p<0.0001)$. This clearly indicates that DBT is markedly superior for detecting subtle masses in complex breast structures.

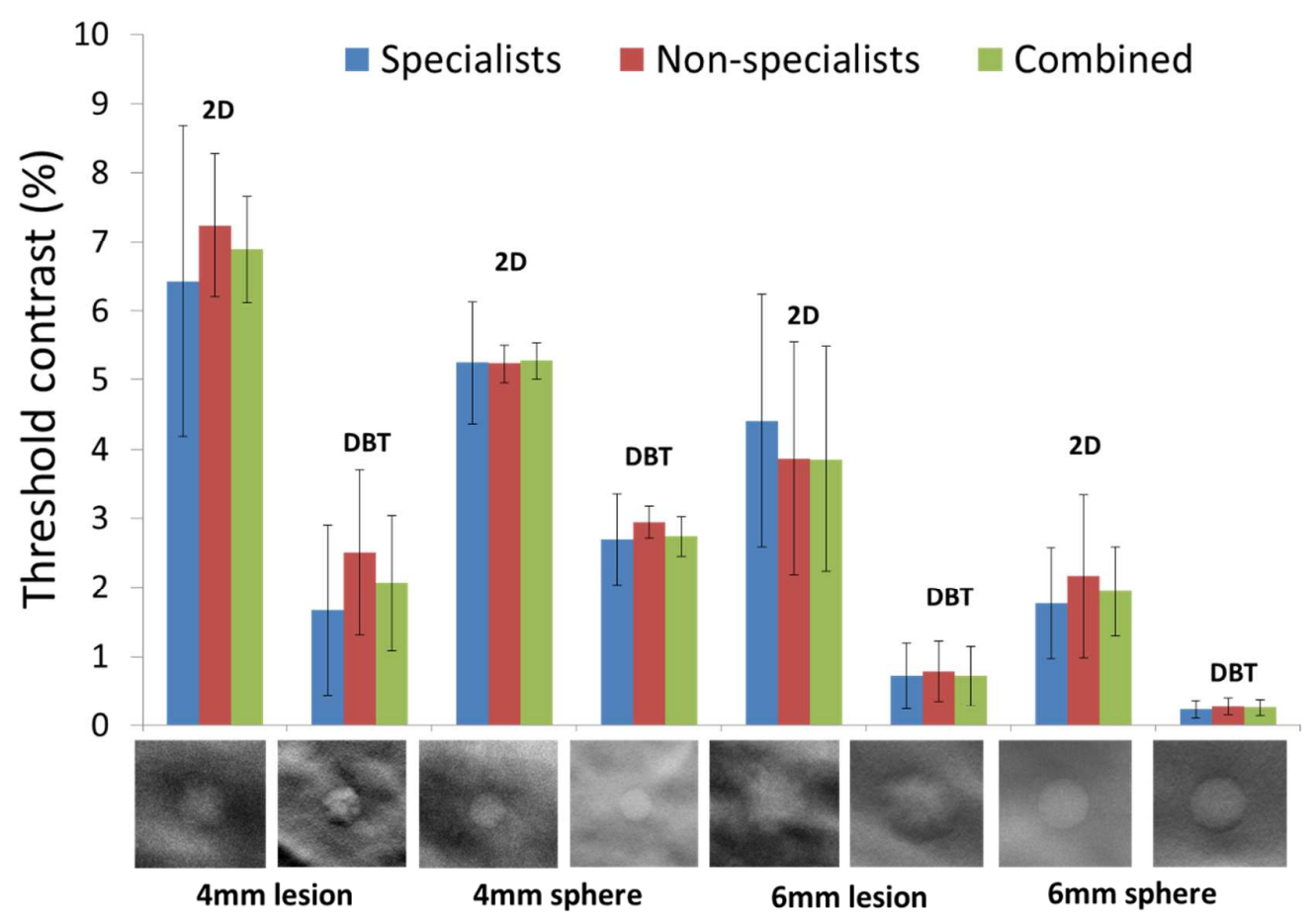


Figure 4 Detectability thresholds for mass and sphere targets for both groups. Errors bars represent $95 \%$ confidence intervals.

By contrast, our previous study (Hadjipanteli et al 2016) indicated that microcalcifications clusters can be more reliably detected in 2D than in DBT systems (both narrow-angle and wide-angle). This is attributed to superior spatial resolution, enhanced dose per projection and zero X-ray tube motion blur in 2D compared to the DBT systems simulated, rendering 2D superior for detecting point-like high contrast objects. This raises questions for optimising DBT detection performance in screening.

Both the combined (average response) and individual observer groups found spherical target detection easier than mass targets detection, as evidenced by the lower contrast threshold for spheres compared to masses. The threshold contrast for $4 \mathrm{~mm}$ spheres was 1.3 times lower than the mass lesion counterparts for $2 \mathrm{D}(p<0.0001)$. With $6 \mathrm{~mm}$ spheres, the differences in contrast threshold were much lower for both $2 \mathrm{D}(\times 2)$, and DBT $(\times 2.7)$ compared to the irregular mass targets of similar size $(p<0.0001)$. In both cases, this is attributed to the presence of high contrast regular edges for spherical targets, as well as their uniform composition, which is perceptually easier to resolve, even when only a limited portion of the target is visible. Thus, a uniform object with regular edges in a complex background appears easier to detect than an irregular object in a complex background. This supports the importance of target/background choice for inferring clinically relevant results. The only exception was for $4 \mathrm{~mm}$ objects and DBT, where the observers found irregular masses easier to detect than spheres resulting in low threshold contrast for masses than spheres $(p<0.0001)$. This result is consistent with our previous study where $3 \mathrm{~mm}$ spheres and masses were used in a 4AFC study (Elangovan et al 2015). This may be because the effect of background texture on object appearance is small for very small objects, particularly in DBT, and as a result irregular objects with irregular edges would have been more easily detectable than regular objects such as spheres.

Table 2 Reading times for two groups with 95\% confidence intervals for 2D and DBT

\begin{tabular}{ccccc}
\hline & \multicolumn{2}{c}{ 2D } & DBT \\
\cline { 2 - 5 } & Specialist & Non-specialist & Specialist & Non-specialist \\
\hline Masses $(4 \mathrm{~mm})$ & $4.8 \mathrm{~s}[4.5,5.1]$ & $10.1 \mathrm{~s}[9.1,11.1]$ & $5.4 \mathrm{~s}[4.9,5.8]$ & $8.6 \mathrm{~s}[7.8,9.3]$ \\
Spheres $(4 \mathrm{~mm})$ & $5.4 \mathrm{~s}[4.9,5.8]$ & $10.3 \mathrm{~s}[8.4,12.1]$ & $7.6 \mathrm{~s}[6.5,8.6]$ & $9.9 \mathrm{~s}[8.5,11.3]$ \\
Masses $(6 \mathrm{~mm})$ & $5.4 \mathrm{~s}[5.0,5.8]$ & $5.9 \mathrm{~s}[5.2,6.5]$ & $4.5 \mathrm{~s}[4.1,5.0]$ & $4.3 \mathrm{~s}[3.7,4.9]$ \\
Spheres $(6 \mathrm{~mm})$ & $4.0 \mathrm{~s}[3.5,4.5]$ & $4.5 \mathrm{~s}[3.6,5.3]$ & $3.9 \mathrm{~s}[2.9,4.9]$ & $4.5 \mathrm{~s}[4.1,5.0]$ \\
\hline
\end{tabular}

In the second arm of the experiment the performance of specialist observers (medical physicists and clinical readers) and non-specialist observers in 4AFC observer studies was considered. There was no statistically significant difference $(p>0.05)$ between specialist and non-specialist groups for the $4 \mathrm{~mm}$ sphere (2D), $6 \mathrm{~mm}$ lesion (2D), $6 \mathrm{~mm}$ lesion (DBT) and $6 \mathrm{~mm}$ sphere (DBT) targets. However there was a significant difference in observer performance $(p<0.05)$ between the two groups favouring the specialist observers for the $4 \mathrm{~mm}$ mass (2D), $4 \mathrm{~mm}$ mass (DBT), $4 \mathrm{~mm}$ sphere (DBT) and $6 \mathrm{~mm}$ sphere (2D) targets. These data present a mixed picture with apparently some experimental conditions favouring one group over another. Overall, non-specialist observers reached the same conclusions as 
specialist observers in comparing differences in 2D and DBT imaging, albeit at a slightly higher threshold contrast (mean difference approx. 16\%, ranging from $0.3 \%$ to $34 \%$ )

There was a significant difference between the reading times between these groups for $4 \mathrm{~mm}$ targets in both $2 \mathrm{D}$ and DBT $(p<0.0001)$. In the case of $2 \mathrm{D}$ images with $4 \mathrm{~mm}$ targets, non-specialists required twice the reading time compared to specialists, representing a 5-6 second time difference for each set of image trials. For the DBT data, the reading time difference narrowed to approximately 3 seconds, with a ratio of 1.4 in reading times for both types of target. As the non-specialists saw $2 \mathrm{D}$ data before the DBT data, it may be that this narrowing represents an increasing familiarity and confidence with the task leading to faster decision speeds for the non-specialists. No significant difference $(p>0.05)$ in reading times for $6 \mathrm{~mm}$ targets was seen by the observers after viewing $4 \mathrm{~mm}$ targets, which supports this notion. It remains to be seen if parity of reading time between both groups could be achieved once non-specialist observers gain a certain level of 4AFC experience. This might facilitate use of 'citizen observers' in some studies (Yeotikar et al 2013).

Table 3 P-values (t-test) for contrast detection thresholds and reading times between two groups (specialist and non-specialist groups)

\begin{tabular}{lcccc}
\hline & \multicolumn{2}{c}{ Contrast detection threshold } & \multicolumn{2}{c}{ Reading times } \\
\cline { 2 - 5 } & 2D & DBT & 2D & DBT \\
\hline Masses (4mm) & $<0.0001$ & $<0.0001$ & $<0.0001$ & $<0.0001$ \\
Spheres (4mm) & 0.20 & $<0.0001$ & $<0.0001$ & $<0.0001$ \\
Masses (6mm) & 0.07 & 0.09 & 0.37 & 0.32 \\
Spheres (6mm) & $<0.0001$ & 0.08 & 0.17 & 0.46 \\
\hline
\end{tabular}

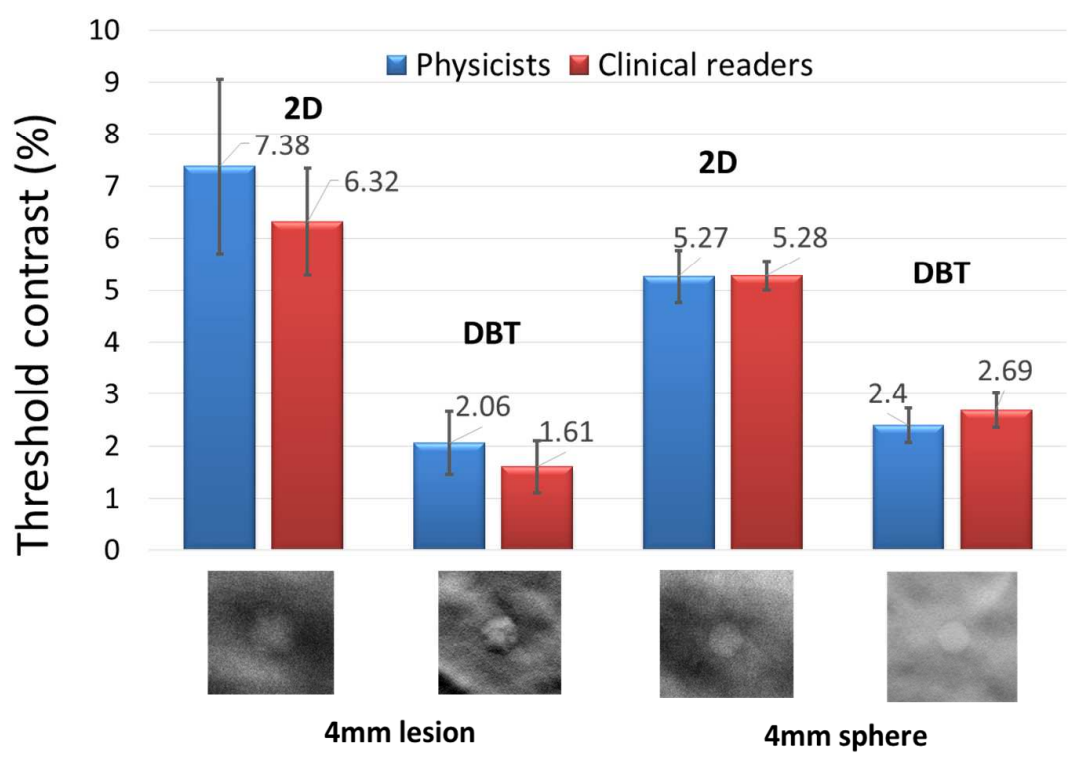


Figure 5 Detectability thresholds for mass and sphere targets for mammography clinical readers and physicists. Errors bars represent $95 \%$ confidence intervals.

Figure 5 examines the 4AFC performance of the two specialist observer groups - clinical readers and medical physicists. The error bars show 95\% confidence intervals. The reading times for the two specialist subgroups are presented in Table 4 . The results of the statistical test for both contrast detection thresholds and reading times for specialist subgroups are shown in Table 5. Further analysis of the results for the specialist observers revealed a significant difference $(p<0.0001)$ in detection performance for mass targets between physicists and clinical readers. This might be attributed to the clinical readers' experience with screen detection as they may be more sensitive to mass-like objects. This diversity may explain the large error bars seen in Figure 4 for the specialist observers. However, there was no significant difference $(p>0.05)$ in detection performance between the two specialist subgroups for spherical objects, nor in reading times between two specialist subgroups, although clinical readers marginally outperformed physicists.

Table 4 Reading times for two specialist sub-groups (clinical readers and physicists) with $95 \%$ confidence intervals for $2 \mathrm{D}$ and DBT

\begin{tabular}{ccccc}
\hline & \multicolumn{3}{c}{ 2D } & DBT \\
\cline { 2 - 5 } & Physicists & $\begin{array}{l}\text { Mammography } \\
\text { clinical readers }\end{array}$ & Physicists & $\begin{array}{l}\text { Mammography } \\
\text { clinical readers }\end{array}$ \\
\hline Masses $(4 \mathrm{~mm})$ & $4.8 \mathrm{~s}[4.5,5.1]$ & $4.9 \mathrm{~s}[4.5,5.1]$ & $6.0 \mathrm{~s}[5.3,6.5]$ & $5.4 \mathrm{~s}[4.9,5.8]$ \\
Spheres $(4 \mathrm{~mm})$ & $5.8 \mathrm{~s}[5.1,6.5]$ & $5.4 \mathrm{~s}[4.7,6.0]$ & $8.3 \mathrm{~s}[6.5,10.2]$ & $7.5 \mathrm{~s}[6.5,8.6]$ \\
\hline
\end{tabular}

Table 5 P-values (t-test) for contrast detection thresholds and reading times between two specialist groups (clinical readers and physicists)

\begin{tabular}{lcccc}
\hline & \multicolumn{2}{c}{ Contrast detection threshold } & \multicolumn{2}{c}{ Reading times } \\
\cline { 2 - 5 } & 2D & DBT & 2D & DBT \\
\hline Masses (4mm) & $<0.0001$ & $<0.0001$ & 0.67 & 0.23 \\
Spheres (4mm) & 0.55 & 0.06 & 0.22 & 0.37 \\
\hline
\end{tabular}

This study has quantitatively explored the difference between different breast imaging modalities and observer cohorts. Of course the study is not without its limitations. Clinically, breast masses occur in different shapes and sizes, here we used mass models of only two sizes $(4 \mathrm{~mm}$ and $6 \mathrm{~mm})$ in order to control the experimental conditions. The density of the masses were assumed to be same as the glandular tissue; this assumption was based on the limited data available in Hammerstein et al (1979). As a result, the low contrast masses were simulated by placing the simulated masses in the high glandular region of the breast model. The breast model represents only a limited range of patterns typically found in real world clinical breast data (Elangovan et al 2017). Furthermore, the breast models used for the study were all $6 \mathrm{~cm}$ thick and were simulated with a breast density of $20 \%$ by volume, based on an analysis of a sample of mammograms from our screening centres. Such breasts might be considered as being quite dense (e.g. Brandt et al 2016) and it could be that differences in 
contrast threshold between mammography and DBT would be higher for dense breasts and lower for very fatty breasts. This can be considered a limitation of the present study. Future work is needed to expand our study to include different breast thicknesses and densities. This will aid in the better understanding of how variability in patients affects the 2D and DBT detection performance.

4AFC studies are predominantly used to quantitatively establish the effectiveness of an imaging modality. These are detection studies that do not involve search or radiological interpretation, but are a useful for understanding the relative importance of imaging techniques. The results of 4AFC studies should be used alongside data from more clinically relevant studies using human subjects, when advising on imaging techniques in mammography. This study was undertaken for only one type of imaging system and so the comparison between DBT and 2D are only relevant to Hologic Dimensions system. However comparison between different observer cohorts can be assumed to be valid for any system.

\section{Conclusions}

In summary, the results of this 4AFC study using the OPTIMAM VCT toolbox has quantified the extent to which DBT is superior for detecting masses compared to 2D-mammography, as demonstrated by lower contrast thresholds in all target categories (2-8 times lower than 2D). This also demonstrates the VCT paradigm as offering a fast and well-controlled alternative method to clinical trials for rapid evaluation and comparison studies. The consistent difference in threshold contrast between spheres and mass lesions (mean difference approx. $42 \%$, ranging from $23 \%$ to $63 \%$ ) demonstrates that such uniform geometric objects are systematically easier to detect across both $2 \mathrm{D}$ and DBT imaging modalities. Furthermore, the findings of this study support the use of non-specialist observers (with training) supplementing the typically limited local pool of specialist observers for certain AFC tasks. This may facilitate larger numbers of observers in such studies to reduce statistical uncertainties.

\section{Acknowledgements}

This work is part of the OPTIMAM2 project funded by Cancer Research UK (grant number: C30682/A17321). We are grateful to Hologic for the provision of the image processing and reconstruction software. We thank colleagues at NCCPM and breast readers at the Jarvis Breast Screening Centre, Guildford for participating in the observer study.

\section{References}

Bakic P R, Albert M, Brzakovic D and Maidment A D A 2002a Mammogram synthesis using a 3D simulation. II. Evaluation of synthetic mammogram texture Med. Phys. 29, 2140-2151.

Bakic P R, Albert M, Brzakovic D and Maidment A D A 2002b Mammogram synthesis using a 3D simulation. I. Breast tissue model and image acquisition simulation Med. Phys. 29, 2131-2139.

Boone J M, Fewell T R \& Jennings R J 1997 Molybdenum, Rhodium and Tungsten anode spectral models using interpolating polynomials with application to mammography Med. Phys. 24, 18631874.

Biederman, I 1987 Recognition-by-components: A theory of human image understanding Psychological Review 94, 115-147. 
Bliznakova K, Sechopoulos I, Buliev I and Pallikarakis N 2012 BreastSimulator: A Software Platform for Breast X-ray Imaging Research J. Biomed. Graph. Comp. 2, 1-14.

Brandt K R, Scott C G, Ma L, Mahmoudzadeh A P, Jensen M R, Whaley D H, Wu F F, Malkov S, Hruska C B, Norman A D, Heine J, Shepherd J, Pankratz V S, Kerlikowske K \& Vachon C M 2016 Comparison of Clinical and Automated Breast Density Measurements: Implications for Risk Prediction and Supplemental Screening Radiology 279, 710-719.

Burgess A E 1995 Comparison of receiver operating characteristic and forced choice observer performance measurement method Med. Phys. 22 643-55

Burgess A E 1999 Mammographic structure: Data preparation and spatial statistics analysis Proc. SPIE. 3661, 642-653.

Chakraborty D P, Breatnach E S, Yester M V, Soto B, Barnes G T and Fraser R G 1986 Digital and conventional chest imaging: a modified ROC study of observer performance using simulated nodules Radiology 158, 35-39.

Ciatto S, Houssami N, Bernardi D, Caumo F, Pellegrini M, Brunelli S, Tuttobene P, Bricolo P, Fantò C, Valentini M, Montemezzi S and Macaskill P 2013 Integration of 3D digital mammography with tomosynthesis for population breast-cancer screening (STORM): a prospective comparison study. Lancet Oncology 14, 583-589.

Dance D R, Young K C and van Engen R E 2011 Estimation of mean glandular dose for breast tomosynthesis: factors for use with the UK, European and IAEA breast dosimetry protocols Phys. Med. Biol. 56, 453-471.

Diaz O, Dance D R, Young K C, Elangovan P, Bakic P R \& Wells K 2014 A fast scatter field estimator for digital breast tomosynthesis Phys. Med. Biol. 59(15), 2375-2390.

Diekmann F \& Bick U 2007 Tomosynthesis and contrast-enhanced digital mammography: recent advances in digital mammography Eur. Radiol. 17, 3086-3092.

Elangovan P, Warren L M, Mackenzie A, Rashidnasab A, Diaz O, Dance D R, Young K C, Bosmans H, Strudley C J \& Wells K 2014 Development and validation of a modelling framework for simulating 2D-mammography and breast tomosynthesis Phys. Med. Biol. 59(15), 4275-4293.

Elangovan P, Rashidnasab A, Mackenzie A, Dance D R, Young K C, Bosmans H, Segars W P and Wells K 2015 Performance comparison of breast imaging modalities using a 4AFC human observer study Proc. SPIE. 9412, 94121T.

Elangovan P, Hadjipanteli A, Mackenzie A, Dance DR, Young KC \& Wells K 2016 OPTIMAM image simulation toolbox - recent developments and ongoing studies Proc. IWDM 9699, 668-675.

Elangovan P, Mackenzie A, Dance D R, Young K C, Cooke V, Wilkinson L, Given-Wilson R M, Wallis M G and Wells K 2017 Design and Validation of Realistic Breast Tissue Models for Use in Virtual Clinical Trials Phys. Med. Biol. 62, 2278-2794.

Gilbert F J, Tucker L and Young K C 2016 Digital Breast Tomosynthesis (DBT): A review of the evidence for use as a screening tool Clinical Radiology 71, 141-50.

Gong X, Glick S J, Liu B, Vedula A A and Thacker S 2006 A computer simulation study comparing lesion detection accuracy with digital mammography, breast tomosynthesis, and cone-beam CT breast imaging Med. Phys.33, 1041-1052.

Graff C G 2016 A new open-source multi-modality digital breast phantom Proc SPIE. 9783, 978309.

Hadjipanteli A, Elangovan P, Mackenzie A, Looney P T, Wells K, Dance D R \& Young K C 2016 The effect of system geometry and dose on the threshold detectable calcification diameter in 2Dmammography and digital breast tomosynthesis Phys. Med. Biol. 62(3), 858-877.

Hammerstein G R, Miller D W, White D R, Masterson M E, Woodard H Q \& Laughlin J S 1979 Absorbed radiation dose in mammography Radiology 130 485-91 (1979).

Hevie M A, Chang J T, Hendrick R E \& Banerjee M 2014 Reduction in late-stage breast cancer incidence in the mammography era: Implications for overdiagnosis of invasive cancer. Cancer 120(17), 2649-2656. 
Highnam R, Brady M, Yaffe M, Karssemeijer N \& Harvey J 2010 Robust breast composition measurement Volpara ${ }^{\mathrm{TM}}$ Proc. IWDM '10, 342-344.

Huda W, Ogdena K M, Scalzettia E M, Parka J, Hunt R \& Dance D R 2003 How does lesion location affect detection performance in digital mammography? Proc SPIE. 5034, 242-252.

Huda W, Ogdena K M, Scalzettia E M, Dudley E F, Dance D R 2004 How do radiographic techniques affect mass lesion detection performance in digital mammography? Proc SPIE. 5372, 372-382.

Huda W, Ogden K M, Scalzetti E M, Dance D R \& Bertrand E A 2006 How do lesion size and random noise affect detection performance in digital mammography? Acad. Radiol. 13(11), 13551366.

ICRU 1992 Photon, electron, proton and neutron interaction data for body tissues Tech. Rep. International Commission on Radiation Units and Measurements 46.

Karim-Kos H E, de Vries E, Soerjomataram I, Lemmens V, Siesling S \& Coebergh J W 2008 Recent trends of cancer in Europe: A combined approach of incidence, survival and mortality for 17 cancer sites since the 1990s European Journal of Cancer 44(10), 1345-1389.

Lai C, Liu X, You Z, Shen Y, Zhong Y, Chen L, Han T, Ge S, Yi Y, Geiser W R, Flores D, Whitman G J, Yang W T \& Shaw1 C C 2010 Detection of simulated microcalcifications in digital mammography effects of quantum and anatomic noises: preliminary study Proc. SPIE. 7622, $76224 \mathrm{Y}$.

Li C M, Segars W P, Tourassi G D, Boone J M and Dobbins J T 2009 Methodology for generating a 3D computerized breast phantom from empirical data Med. Phys. 36, 3122-3131.

Mackenzie A, Dance D R, Workman A, Yip M, Wells K \& Young K C 2012 Development and validation of a method for converting images to appear with noise and sharpness characteristics of a different detector and X-ray system Med. Phys. 39, 2721-2734.

Mackenzie A, Dance D R, Diaz O \& Young K C 2014 Image simulation and a model of noise power spectra across a range of mammographic beam qualities Med. Phys. 41, 12901-14.

Macmillan N A and Creelmans C D 2004 Detection theory, A user's guide, 2nd edition, Lawrence Erlbaum Associates Inc

Maidment A D A 2014 Virtual clinical trials for the assessment of novel breast screening modalities. Proc IWDM '14. 8539, 1-8.

Mie'ville F A, Gudinchet F, Brunelle F, Bochud F O \& Verdun F R 2013 Iterative reconstruction methods in two different MDCT scanners: Physical metrics and 4-alternative forced-choice detectability experiments - A phantom approach Physica Medica 29, 99-110

Niklason et al 1997 Digital tomosynthesis in breast imaging Radiology 205, 399-406 (1997).

Sandborg U M, Hunt R, Dance D R \& Alm Carlsson G 2003 Implementation of pathologies in the Monte Carlo model in chest and breast imaging. Tech. Rep. Institutionen för radiologi, Universitetet $i$ Linköping 94.

Shaheen E, Zanca F, Sisini F, Zhang G, Jacobs J \& Bosmans H 2010 Simulation of 3D objects into breast tomosynthesis images Radiat. Prot. Dosim. 139, 108-112.

Shams L and von der Malsburg 2002 Acquisition of visual shape primitives Vision Research 42, 2105 2122.

Siddon R L 1985 Fast calculation of the exact radiological path for a three-dimensional CT array Med. Phys. 12, 252-255.

Skaane P, Bandos A I, Gullien R, Eben E B, Ekseth U, Haakenaasen U, Izadi M, Jebsen I N, Jahr G, Krager M, Niklason L T, Hofvin S \& Gur D 2013 Comparison of digital mammography alone and digital mammography plus tomosynthesis in a population-based screening program Radiology 267, 652-660.

Rashidnasab A, Elangovan P, Yip M, Young K C, Dance D R \& Wells K 2013a Simulation and assessment of realistic breast lesions using fractal growth models Phys. Med. Biol. 15, 5613-5626. 
Rashidnasab A, Elangovan P, Diaz O, Mackenzie A, Young K C, Dance D R \& Wells K 2013b Simulation of 3D DLA masses in digital breast tomosynthesis Proc. SPIE. 8668, 86680Y.

Rashidnasab A, Bemelmans F, Marshall N W, Kimpe T \& Hilde Bosmans 2016 Impact of Clinical Display Device on Detectability of Breast Masses in 2D Digital Mammography: A Virtual Clinical Study Proc. IWDM 9699, 676-683.

Timberg P, Lång K, Nyström M, Holmqvist K, Wagner P, Förnvik D, Tingberg A and Zackrisson S 1987 Investigation of viewing procedures for interpretation of breast tomosynthesis image volumes: a detection-task study with eye tracking Eur Radiol. 23, 997-1005

Timberg P, Båth M, Andersson I, Mattsson S, Tingberg A \& Ruschin M 2012 Visibility of microcalcification clusters and masses in breast tomosynthesis image volumes and digital mammography: 4AFC human observer study Med. Phys. 39 (5), 2431-2437.

Wallis M G, Moa E, Zanca F, Leifland K \& Danielsson M 2012 Two-view and single-view tomosynthesis versus full-field digital mammography : high-resolution X-ray imaging observer study Radiology 262, 788-796.

Yeotikar N S, Khuu S K, Asper L J \& Suttle C M 2013 Context and Crowding in Perceptual Learning on a Peripheral Contrast Discrimination Task: ContextSpecificity in Contrast Learning Plos One 8(5), 1-8.

Young S, Bakic P R, Myers K J, Jennings R J \& Park S 2013 A virtual trial framework for quantifying the detectability of masses in breast tomosynthesis projection data Med. Phys. 40(5), 051914:10519141:15. 